\title{
Application of Fourier Transform Infrared Spectroscopic Imaging to Biomedical Analyses
}

Rohit Bhargava, Daniel C. Fernandez, Scott W. Huffman, Michael D. Schaeberle and Ira W. Levin

Laboratory of Chemical Physics, Building 5, Room B1-38, National Institute of Diabetes and Digestive and Kidney Diseases, National Institutes of Health, Bethesda, MD 20892-0510 USA

Traditionally, tissue characterization and disease diagnosis are based on subjective morphological examination. As histopathological abnormalities in large tissues often arise from chemical changes in localized areas, a thorough chemical investigation of histopathologic foci is possible using microspectroscopic techniques, for example, Fourier transform infrared microspectroscopy. However, some challenges needed to be overcome before FTIR microspectroscopy could be applied to study tissue biopsies. In particular, the technique suffered from poor spatial resolution and poor signal to noise ratio (SNR) characteristics. The lack of wavelength-limited spatial resolution capable instrumentation and the poor SNR characteristics reduced confidence in the quantification of spectral changes from small, localized areas. Further, the rate of data acquisition was not rapid enough to allow examination of a large number of samples in a short time. Recent advances in infrared microspectroscopy have led to alleviation of many of these issues. In particular, the concept of obtaining FTIR spectroscopic measurements by coupling multichannel detectors mounted on infrared microscopes to infrared interferometers [1] allows us to obtain spectroscopic information from small spatial regions faster than ever before. Data obtained in a multichannel modality combined with advances in detector technology offers significant advantages in acquiring large amounts of data in a short time [2].

FTIR spectroscopic imaging of tissues allows us to simultaneously obtain morphological and chemical information to characterize normal and diseased states for different types of tissues. We present examples illustrating these developments by presenting examples of histopathological determinations in skin, prostate and esophageal tissue sections. Chemical differences in these complex tissue sections are readily identified from images based upon the spectral absorbance characteristics of specified infrared vibrational modes, which allows for identification akin to regular histological analyses. Spectral differences from localized regions, as in figure 1 below, allows for the characterization of lesions. In this case, we examine the spectral signature of lesions indicative of Birt-Hogg-Dube Syndrome, which is a marker for kidney neoplasia [3]. We present examples of other tissue biopsies that show localized chemical differences between normal and diseased states including thin sections from prostatic tissue and esophageal tissue biopsies.

\section{References}

[1] E.N. Lewis et al., Anal. Chem. 67 (1995) 3377.

[2] R. Bhargava and I.W. Levin, Anal. Chem. 73 (2001) 5157.

[2] J.R. Toro et al., Arch. Dermatol. 135 (1999) 1195 -1202. 


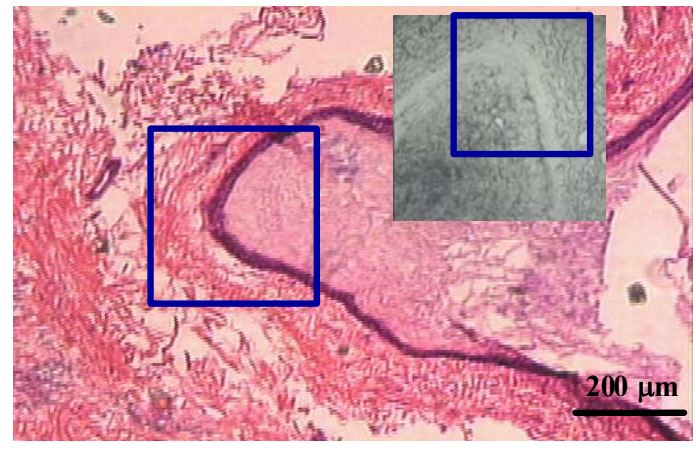

H\&E Stained Image

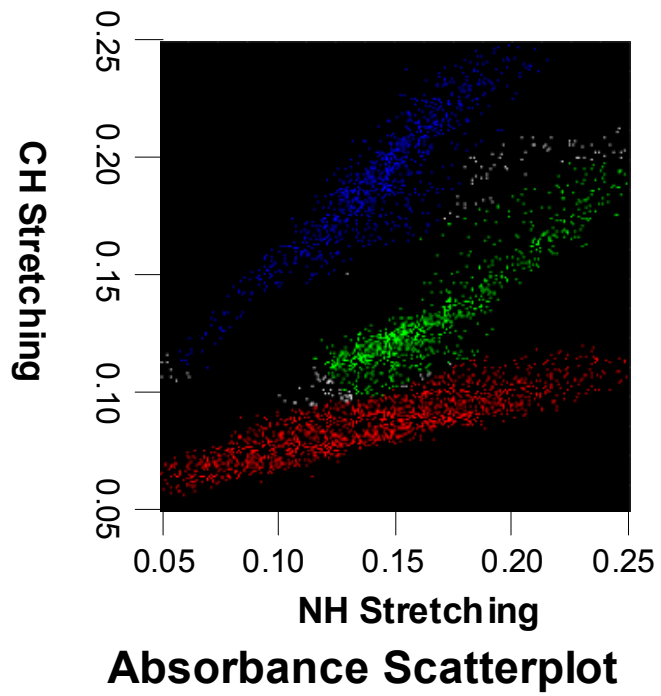

NH Stretching CH Stretching

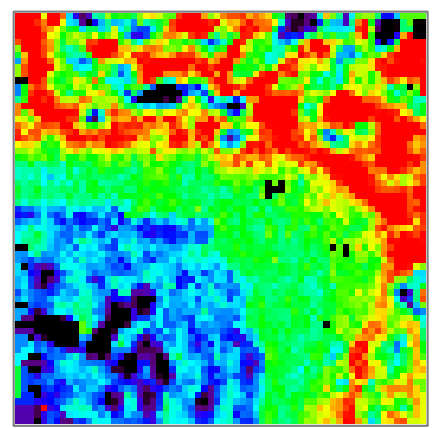

Low

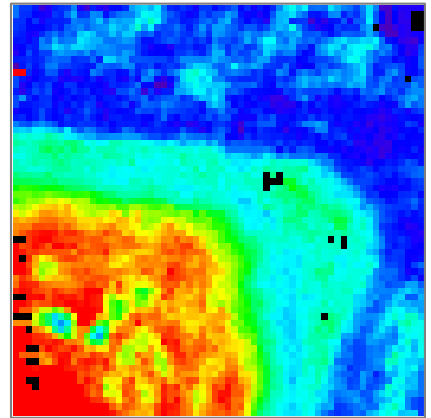

High

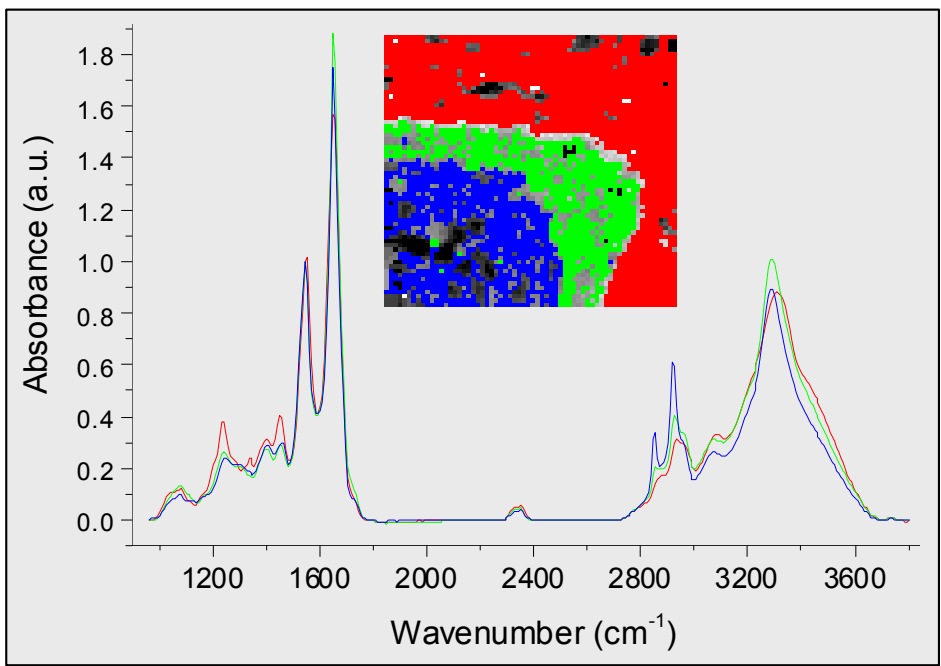

Figure 1. A Hematoxylin and Eosin (H\&E) stained image of a skin biopsy (top, left). The inset in the top left figure shows a subsequent section of the tissue that has not been stained. The infrared images at specific absorption modes (top, right) corresponding to the boxed region of the unstained image show differences in infrared intensity. A scatter plot of all pixels (bottom, left) reveals three distinct regions with their characteristic absorbance spectra (bottom, right), which are shown baseline corrected and normalized to the intensity of the Amide II stretching vibrational mode. The inset shows the color coded regions obtained from the scatter plot. 